\title{
Variaciones de tres índices larvarios de Aedes aegypti (L.) (Diptera: Culicidae) y su relación con los casos de dengue en Yurimaguas, Perú, 2000 - 2002
}

\author{
WERTHER FERNANDO FERNÁNDEZ* y JOSÉ IANNACONE*
}

\section{VARIATIONS OF THREE Aedes aegypti (L.) LARVAL INDEXES (DIPTERA: CULICIDAE) AND ITS RELATIONSHIP WITH DENGUE CASES IN YURIMAGUAS, PERU, 2000-2002}

A research about population behaviour of Aedes aegypti (L.) in its larval phase, to explain their fluctuations through three entomological indexes and their relationships with dengue cases in Yurimaguas city, Loreto, Peru was performed. Positive housing were surveyed through three Entomological Indexes (EI) \{Index of Domiciliary Infestation or Housing Index or also named Aedic Index (AI), Container Index (CI) or Breteu Index (BI)\} and the kind of container more common in 11 zones of Yurimaguas city (5 53'30" L.S; $76^{\circ} 05^{\prime} 26^{\prime \prime}$ L.O), Loreto, Peru during 2000 to 2002. The three entomological indexes showed differences between all twelve evaluations performed during 2000 to 2002. In the three EI had observed a tendency to decrease from April-2000 to December2002. AI decreased in $46.82 \%$; CI in $67.29 \%$ and IB decreases in $43.71 \%$. The cases of dengue registered among 2000 to 2002 decreased $80.6 \%$. None differences between 11 zones in Yurimaguas for AI, CI and BI were observed. The three EI: AI, BI and CI all had highly lineal correlation and positive during all period 2000 to 2002. Only in AI and dengue cases, were found lineal correlation. In some evaluations among 2000 to 2002 existed significantly differences between ten categories of containers in CI and BI. Higher values for the next four categories: 10 (useless), 9 (flower pot and flower pot stand), 6 (rubber tyre), three (barrel, cylinder, sanson) were observed. The variations of EI during wet and dry periods were evaluated.

Key words: Aedes, Dengue, Entomological indexes, Peru.

\section{INTRODUCCIÓN}

Las enfermedades transmitidas por insectos vectores son un importante problema de salud en el mundo ${ }^{1}$. En el Perú, en la última década, se ha incrementado el número de casos de enfermedades transmitidas por ellos principalmente por tres factores: la introducción de personas a zonas endémicas, la modificación indiscriminada del medio ambiente, y el no tratamiento o tratamiento incompleto de las personas. Este incremento implica un alto costo social y económico, especialmente porque la población expuesta es la de menos recursos y usualmente se encuentra en zonas deprimidas ${ }^{2}$.

Una de las principales enfermedades

* Laboratorio de Ecofisiología Animal. Facultad de Ciencias Naturales y matemáticas. Universidad Nacional Federico Villarreal. Calle San Marcos 383, Lima - Perú. E-mail: joseiannacone@gmail.com 
transmitidas por vectores es la enfermedad viral conocida con el nombre de dengue, considerada por la Organización Mundial de la Salud (OMS) como una enfermedad emergente ${ }^{3-7}$. El dengue clásico y el hemorrágico vienen constituyendo un serio problema de Salud Pública y un problema creciente cada vez más grave para los países americanos, en especial para el Perú, que se han visto afectados en los últimos años por extensas y explosivas epidemias, repercutiendo negativamente en las economías de los países ${ }^{8-11}$. Origina grandes gastos, derivados por la hospitalización de los pacientes y/o por las necesarias campañas de control de su vector, el Aedes aegypti ${ }^{4-12-18}$.

A partir del reingreso de su vector A. aegypti en el año 1984 en la Región de Loreto y Ucayali del Perú, el dengue se dispersó a nuevas zonas de tal manera que ya se han descrito casos en trece departamentos del Perú ${ }^{19-20}$.

El estudio de las fluctuaciones larvarias de $A$. aegypti, a través de Indicadores Entomológicos (IE) posibilitan la definición y/o reorientación de estrategias de control permitirán disminuir el contacto vector - hombre ${ }^{21-23}$. Para que esto suceda, es necesario realizar estudios para determinar los lugares en donde se reproducen y la manera como contribuyen a la transmisión $\mathrm{n}^{6,10,24,25}$.

Las metodologías más comunes emplean los muestreos de larvas de los Aedes en vez de las recolecciones de huevos o de adultos. La unidad básica de muestreo es la casa o inmueble la que se inspecciona sistemáticamente en búsqueda de depósitos o recipientes que contengan agua. En éstas, se busca larvas y pupas de mosquitos y sus exuvias ${ }^{26}$. Normalmente se emplean tres índices para registrar los niveles de infestación: el Índice de Infestación domiciliaria o Índice aédico (IA), el Índice de recipientes (IR) y el Índice de Breteau (IB) $)^{5,15,27}$.

El presente estudio tratará de explicar las fluctuaciones poblacionales del A. aegypti en su fase larvaria a través de estos tres IE y su relación con los casos de dengue en la ciudad de Yurimaguas, Loreto, Perú en los años 2000 al 2002. A la vez se estudiará estos índices en diez categorías diferentes de recipientes o contenedores de agua.

\section{MATERIAL Y MÉTODOS}

Ámbito del estudio. Yurimaguas, capital de la provincia de Alto Amazonas, se ubica en el extremo Nor-Oeste de la Amazonía Peruana, en la confluencia de los ríos Shanusi y Paranapura con el Huallaga (5 $5^{\circ} 3^{\prime} 30^{\prime \prime}$ L.S $76^{\circ} 05^{\prime} 26^{\prime \prime}$ L.O), a una altitud de $182 \mathrm{msnm}$. Se asienta en su mayor parte sobre una terraza alta respecto al río Huallaga, con una topografía relativamente planaaccidentada y atravesada por cuatro quebradas. El clima es húmedo tropical con lluvias frecuentes durante todo el año, pero con dos épocas o periodos bien definidos, el de Noviembre - Abril que es el periodo de mayor incidencia de lluvias (época lluviosa) y el de los meses de Mayo Octubre que es el periodo de menos lluvias (época seca). La precipitación pluvial anual es de 2.200 $\mathrm{mm}$. La temperatura media máxima mensual es de $32,3^{\circ} \mathrm{C}$ y una media mínima mensual de $20,6^{\circ} \mathrm{C}$. La temperatura promedio es de $26^{\circ} \mathrm{C}$. La ciudad es el centro socioeconómico de la provincia y es la segunda ciudad más importante y más poblada de la región Loreto, con 52.000 habitantes, tiene una superficie de $2.684,34 \mathrm{~km}^{2}$, de las cuales $4,16 \mathrm{~km}^{2}$ corresponde al área urbana y los 2.680,16 $\mathrm{km}^{2}$ restantes al área rural. La densidad poblacional no está distribuida de manera uniforme porque el nivel de consolidación es desigual, y en muchos casos, la topografía y las tierras inundadas limitan el crecimiento. La carretera Yurimaguas - Tarapoto facilita la comunicación permanente con la zona norte del país, existiendo un fluido intercambio comercial y desplazamiento poblacional bastante intenso. Asimismo, existe una intensa comunicación fluvial con las ciudades de Iquitos y Pucallpa y demás localidades de la cuenca del Marañón. La ciudad contiene 11 zonas, 322 manzanas y 7.798 viviendas, conformadas en un $61 \%$ urbanas y en un $39 \%$ de su extensión en un área y población urbano-marginal con condiciones bastante precarias de saneamiento ambiental, especialmente en lo que respecta a la provisión de agua y sistema de alcantarillado. Tiene una deficiente aprovisionamiento de agua potable (sólo el $41 \%$ de la población cuenta con conexión domiciliaria), la falta de continuidad en el suministro del agua (algunas zonas sólo tienen agua de la red entre 12 a 14 horas al día) y el deficiente sistema de recolección y eliminación de residuos sólidos y depósitos inservibles (frascos, baldes, otros recipientes etc.), contribuyen a la formación de criaderos de mosquitos intra y peri domicilio. Esto, sumado a las condiciones climáticas, a la accidentada geografía de la ciudad y el flujo constante de personas desde la zona norte y 
oriente del país por el creciente intercambio comercial, convierten a Yurimaguas en una zona de alto riesgo para la transmisión del dengue hemorrágico. El distrito y la ciudad de Yurimaguas, concentra al $42 \%$ (58.627 hab) de la población provincial, es el principal núcleo comercial de la provincia. En este escenario se han presentado los brotes epidémicos de mayor envergadura de la provincia, entre 1997 a 2000.

Diseño del Estudio. Se hizo una exploración diagnóstica de diseño longitudinal y transversal. Solo se incluyó en el análisis para los IE los obtenidos de la vigilancia entomológica al 100\% (censos o intervenciones) realizados en 11 zonas de la ciudad de Yurimaguas durante los años 2000 a 2002. Y sólo para relacionar los IE y los casos de dengue mensuales, se tomaron los datos de los IE al $100 \%$ y paralelamente también los resultados al $10 \%$ y $33 \%$. Los 11 barrios agrupados en zonas fueron: 1) La Favorita, Santa Rosa y La Boca; 2) Moralillos; 3) Aguamiro; 4) Juan Velasco Alvarado, Miguel Irizar Campos, La Dolorosa Virgen de la Natividad; 5) Central; 6) La Loma; 7) La Carretera, La Florida; 8) La Unión, San Juan; 9) Las Flores, Pueblo Joven 82; 10) Las Américas, Ramón Ruiz Hidalgo, Tiwinza; 11) Moisés Tong Lee y General José de San Martín.

Criterios de inclusión. Por problemas operativos encontrados en la vigilancia y el control de A. aegypti se asume que los datos obtenidos desde el año 1996 a Enero de 2000 tienen una gran variabilidad e incongruencia y no son confiables como para someterlos a una evaluación. Debido a esto, en Febrero del 2000, el programa sufre una total reorganización, reorientando las políticas y acciones de vigilancia y control de acuerdo a las guías y manuales referenciales e incorporando nuevas estrategias y componentes como el de participación comunitaria a través del sector Educación con quien se empieza a trabajar en forma conjunta y coordinada, asimismo se cambian los criterios de vigilancia y control. Los datos se obtuvieron de los censos de larvas aédicas realizadas en las inspecciones de viviendas al $100 \%$ en la ciudad durante los años 2000 al 2002. Durante el 2000 se evaluaron en los meses de Abril, Mayo, Junio, Julio, Octubre y Noviembre (no más de 5 zonas por evaluación). En el 2001 en Marzo, Agosto y
Diciembre, y finalmente en el 2002 en Abril, Agosto y Diciembre en todas las zonas.

La determinación de las formas larvarias de A. aegypti se realizó en el Laboratorio de Entomología de la Dirección de Salud Ambiental del Hospital de Apoyo Yurimaguas, Loreto, empleando claves taxonómicas especializadas ${ }^{28-31}$.

Análisis de datos. Se calcularon los siguientes IE para A. aegypti por cada zona y por tipo de recipiente: IID o IA: porcentaje de casas infestadas con larvas de Aedes, $\mathrm{IA}=\left(\mathrm{N}^{\circ}\right.$ de casas positivas/ $\mathrm{N}^{\circ}$ de casas inspeccionadas) 100. IR: porcentaje de depósitos con agua infestados por larvas de Aedes, $\mathrm{IR}=\left(\mathrm{N}^{\circ}\right.$ de recipientes positivos $/$ $\mathrm{N}^{\circ}$ de recipientes inspeccionados) 100 y el IB: número de recipientes positivos por 100 casas inspeccionadas, $\mathrm{IB}=\left(\mathrm{N}^{\circ}\right.$ recipientes positivos/ $\mathrm{N}^{\circ}$ casas inspeccionadas) 100 (OPS 1995).

Se empleó el ANDEVA para: 1) determinar si existían diferencias significativas entre las seis evaluaciones realizadas en el año 2000 (Abril, Mayo, Junio, Julio, Octubre y Noviembre), las tres en el 2001 (Marzo, Agosto y Diciembre) en todas las zonas y las tres en el 2002 (Abril, Agosto y Noviembre) también en todas las zonas, para los tres indicadores: IA, IR e IB; 2) determinar si existían diferencias entre las 11 zonas para el IIA, IR e IB para los años 2001 y 2002. No se consideró el año 2000 para esta prueba, debido a que las evaluaciones no fueron homogéneas durante ese año, pues en las seis evaluaciones mensuales realizadas (Abril, Mayo, Junio, Julio, Octubre y Noviembre 2000) fueron consideradas no más de cinco evaluaciones por mes; 3 ) determinar si existían diferencias entre los IIA, IR e IB de la época seca (Mayo 2000, Junio 2000, Julio 2000, Octubre 2000, Agosto 2001 y Agosto 2002) y lluviosa (Abril 2000, Noviembre 2000, Diciembre 2001, Abril 2002 y Noviembre 2002); 4) determinar si existían diferencias en el IR y el IB entre las diez categorías de recipientes catalogados \{tanque elevado (1), tanque bajopozos (2), barril-cilindros (3), baldes-bateas-tinas (4), ollas (5), llantas (6), canaletas (7), cántaro de barro-jarrón (8), florero-macetas (9) e inservibles (10)\} para cada evaluación y para el total de evaluaciones realizadas entre el 2000 al $2002 ; 5)$ determinar si existían diferencias en el IR e IB para cada evaluación con relación a las once zonas evaluadas para determinar diferencias en el IA, IR e IB entre los años 2000, 2001 y 2002. Posteriormente, se aplicó la prueba post- 
hoc de Tukey para determinar las diferencias entre las evaluaciones llevadas a cabo.

Se realizó una correlación de Spearman $\left(\mathrm{r}_{\mathrm{s}}\right)$ para determinar si existía correlación lineal entre IIA, IR e IB por cada zona evaluada. Para determinar las diferencias entre sector o cono norte (zonas 1 al 5) y sector o cono sur (zonas 6 al 11) de Yurimaguas para los indicadores entomológicos IA, IR e IB se usó la prueba de $t$ de student de muestras independientes. La prueba de $\mathrm{t}$ de student se empleó nuevamente para determinar las diferencias entre el periodo seco y lluvioso entre los tres IE. Para todos los casos se empleó la prueba de homogeneidad de varianzas de Levene como previo requisito para el empleo de las pruebas paramétricas de $t$ de student y el ANDEVA.

Para el cálculo de la estadística descriptiva e inferencial se usó el paquete estadístico SPSS versión 10 para Windows XP.

\section{RESULTADOS}

La Tabla 1 nos indica que el IA presentó diferencias significativas entre las doce evaluaciones durante el 2000 al $2002(\mathrm{~F}=5,71$; g.l. $=11$ y $74 ; \mathrm{P}=0,00)$. Se observó variaciones entre Abril-2000 y Agosto-2001, así como entre Abril-2000 y Diciembre-2002. El IR presentó diferencias significativas entre las doce evaluaciones del 2000 al $2002(\mathrm{~F}=16,89$; g.1. = 11 y 74; $\mathrm{P}=0,00)$, observándose estas entre Abril-2000, Julio-2000 y Agosto-2001, Agosto-
2002 y Diciembre-2002 (Tabla 1). El mismo patrón se presentó para el IB con diferencias estadísticamente significativas entre las doce evaluaciones $(\mathrm{F}=5,56$; g.l. $=11$ y $74 ; \mathrm{P}=0,00)$, principalmente entre Abril-2000 versus Agosto2001 - Diciembre-2002 (Tabla 1). Para estos tres Indicadores entomológicos se ha notado una tendencia a la disminución de Abril-2000 a Diciembre-2002 (Tabla 1).

No se observaron diferencias significativas entre las 11 zonas para el IA y el IB (Tabla 2), aunque existen promedios de IA e IB numéricamente diferentes (compárese zona 2 y zona cuatro para ambos índices), estos son estadísticamente similares debido a las variaciones en la DE. Sólo el IR presentó diferencias entre las zonas cuatro y cinco (Tabla 2). Durante el 2001-2002 no existieron diferencias significativas para el IR de Marzo-2001 a Diciembre-2002 entre las once zonas evaluadas (Tabla 3$)(\mathrm{F}=0,51$ $1,46 ; \mathrm{P}=0,16-0,87)$.

Durante el 2001-2002 no existieron diferencias significativas para IB en Marzo-2001, Agosto2001, Abril-2002 y Agosto-2002 entre las once zonas evaluadas (Tabla 4$)(\mathrm{F}=0,81-2,23 ; \mathrm{P}=$ 0,06-0,62). En cambio Diciembre-2001 presentó diferencias significativas con mayores valores de IB para la zona 1 y en Diciembre-2002 se presentaron valores más altos para la zona once ( $F=1,91-1,94 ; \mathrm{P}=-0,04-0,05)$ (Tabla 4).

La Tabla 5 nos muestra que los tres índices IA, IB e IR estuvieron todos altamente correlacionados lineal y positivamente durante el

Tabla 1. Fluctuación de tres índices entomológicos: IA, IR e IB en doce evaluaciones durante el 2000 al 2002, Yurimaguas, Loreto, Perú

\begin{tabular}{lrrrrrrrrr}
\hline Evaluación & IA $(\mathbf{X}$ & DE $)$ & Sig. & IR $(\mathbf{X}$ & DE $)$ & Sig. & IB $(\mathbf{X}$ & DE $)$ & Sig. \\
\hline Abr-00 & 6,63 & 1,15 & $\mathrm{~b}$ & 4,83 & 1,23 & $\mathrm{~d}$ & 7,74 & 1,06 & $\mathrm{~B}$ \\
May-00 & 5,17 & 3,78 & $\mathrm{ab}$ & 1,25 & 0,98 & $\mathrm{abc}$ & 6,19 & 4,91 & $\mathrm{Ab}$ \\
Jun-00 & 3,19 & 1,04 & $\mathrm{ab}$ & 0,78 & 0,14 & $\mathrm{ab}$ & 3,61 & 0,99 & $\mathrm{Ab}$ \\
Jul-00 & 5,97 & 0,22 & $\mathrm{ab}$ & 2,52 & 0,09 & $\mathrm{c}$ & 7,27 & 0,26 & $\mathrm{Ab}$ \\
Oct-00 & 5,09 & 0,41 & $\mathrm{ab}$ & 2,13 & 0,86 & $\mathrm{bc}$ & 6,15 & 1,30 & $\mathrm{ab}$ \\
Nov-00 & 5,37 & 2,61 & $\mathrm{ab}$ & 1,35 & 0,70 & $\mathrm{abc}$ & 6,31 & 2,97 & $\mathrm{ab}$ \\
Mar-01 & 5,60 & 1,61 & $\mathrm{ab}$ & 1,54 & 0,66 & $\mathrm{abc}$ & 7,28 & 2,51 & $\mathrm{ab}$ \\
Ago-01 & 1,74 & 0,91 & $\mathrm{a}$ & 0,43 & 0,23 & $\mathrm{a}$ & 1,94 & 0,97 & $\mathrm{a}$ \\
Dic-01 & 5,23 & 2,48 & $\mathrm{ab}$ & 1,13 & 0,58 & $\mathrm{abc}$ & 6,28 & 3,06 & $\mathrm{ab}$ \\
Abr-02 & 4,46 & 2,64 & $\mathrm{ab}$ & 1,06 & 0,82 & $\mathrm{ab}$ & 5,83 & 3,67 & $\mathrm{ab}$ \\
Ago-02 & 2,20 & 2,27 & $\mathrm{ab}$ & 0,62 & 0,70 & $\mathrm{a}$ & 2,59 & 2,69 & $\mathrm{ab}$ \\
Dic-02 & 1,63 & 1,43 & $\mathrm{a}$ & 0,40 & 0,29 & $\mathrm{a}$ & 1,93 & 1,70 & $\mathrm{a}$ \\
\hline
\end{tabular}

Letras iguales en una misma columna indican que los promedios son estadísticamente iguales. Sig. = Probabilidad $\mathrm{X}=$ Promedio; $\mathrm{DE}=$ Desviación estándar 
Indices larvarios de Aedes y su relación con dengue cases - W. F. Fernández y J. Iannacone

Tabla 2. Fluctuación de tres índices entomológicos: IA, IR e IB para el 2001 al 2002 por zonas para Yurimaguas, Loreto, Perú

\begin{tabular}{lrrrrrrrrr}
\hline Zonas & IA $(\mathbf{X}$ & DE) & Sig. & IR $(\mathbf{X}$ & DE $)$ & Sig. & IB $(\mathbf{X}$ & DE $)$ & Sig. \\
\hline uno & 4,77 & 3,31 & a & 1,19 & 0,78 & ab & 5,87 & 4,45 & a \\
dos & 5,19 & 2,69 & a & 1,25 & 0,65 & ab & 6,35 & 3,51 & a \\
tres & 2,11 & 2,27 & a & 0,69 & 1,09 & ab & 3,07 & 4,14 & a \\
cuatro & 0,77 & 0,70 & a & 0,21 & 0,23 & A & 1,14 & 1,23 & a \\
cinco & 4,68 & 2,54 & a & 1,57 & 0,99 & B & 5,90 & 3,83 & a \\
seis & 3,32 & 2,21 & a & 0,68 & 0,37 & ab & 3,58 & 2,01 & a \\
siete & 4,50 & 2,73 & a & 0,94 & 0,55 & ab & 5,48 & 3,57 & a \\
ocho & 2,73 & 1,77 & a & 0,57 & 0,35 & ab & 3,28 & 2,23 & a \\
nueve & 2,57 & 1,92 & a & 0,52 & 0,30 & ab & 3,03 & 2,15 & a \\
diez & 3,73 & 2,18 & a & 0,91 & 0,46 & ab & 4,67 & 2,59 & a \\
once & 3,90 & 2,78 & a & 0,96 & 0,84 & ab & 5,02 & 3,91 & a \\
& & & & & & & & & \\
\hline
\end{tabular}

Letras iguales en una misma columna indican que los promedios son estadísticamente iguales. Sig. = Probabilidad.Zonas: 1) La Favorita, Santa Rosa, La Boca. 2) Moralillos. 3) Aguamiro. 4) J. Velasco Alvarado, Miguel Irizar, La Dolorosa, La Natividad. 5) Central. 6) La Loma. 7) La Carretera, La Florida. 8) La Union, San Juan. 9) Las Flores, Pp.Jj. 82. 10) Las Americas, R.Ruiz Hidalgo, Tiwinza. 11) Moises Tong, J. de San Martin.

periodo 2000 al 2002. La Tabla 6 indica para los tres IE una tendencia a la disminución del 2000 al 2002. El IA disminuye en un 46,82\% del 2000 a 2002; el IR, en cambio disminuye en un 67,29 $\%$ del 2000 al 2002 y finalmente IB disminuye en un $43,71 \%$ en el mismo periodo de años. Para los tres IE existen diferencias significativas entre 2000 y 2002 (Tabla 6).

La Tabla 7 señala que los casos de dengue registrados en 36 meses del 2000 al 2002 (que incluyen encuestas al 10\%, 33\% y censos al $100 \%$ ), disminuyeron de 15,5 casos mensuales a $3(80,6 \%)$. Sin embargo, no existieron diferencias significativas entre los tres años de registro, debido a los altos valores en la $\mathrm{DE}(\mathrm{F}=2,88 ; \mathrm{P}=$ 0,07). El mismo patrón se observó al analizar los datos sólo incluyendo las doce evaluaciones al $100 \%$ durante los tres años $(\mathrm{F}=0,36 ; \mathrm{P}=0,70)$; sin embargo, se notó una disminución en un 29,7\%. Además, cuando se llevó a cabo una correlación lineal entre el IA y los casos de dengue, se observa que ambas variables se encuentran correlacionadas linealmente $\left(r_{s}=0,36\right.$; $\mathrm{N}=36 ; \mathrm{P}=0,03) \quad$ La ecuación de regresión lineal $(\mathrm{F}=4,10 ; \mathrm{P}=0,05)$ y la ecuación de regresión cúbica fueron significativas $(\mathrm{F}=8,87$; $\left.\mathrm{P}=0,00 ; \mathrm{r}^{2}=0,45\right)$.

No existió variación en los índices IA, IR e IB entre los sectores o cono Norte (zonas 1 a 5) y Sur (zonas 6 a 11) (Tabla 8). Aunque numéricamente se observa índices mayores para la zona Sur.
Durante el 2000 no existieron diferencias significativas para el IR de Abril-2000 a Julio2000 entre las diez categorías de recipientes (Tabla 9); a excepción de Octubre-2000 en donde se notaron valores más altos de IR entre la categoría 6 (llantas) y el resto de recipientes; también en Noviembre-2000, se observaron diferencias entre la categoría 2 (tanque bajo y pozos) y 9 (florero y macetero) $(\mathrm{F}=4,73 ; \mathrm{P}=$ 0,00). Durante el 2000 existieron diferencias significativas para IB de Abril-2000 a Noviembre2000 entre las diez categorías de recipientes (Tabla 10); y se observó mayormente diferencias con la categoría 10 (inservibles) $(\mathrm{F}=3,73-17,29$; $\mathrm{P}=0,00-0,007)$.

La Tabla 11 muestra la fluctuación existente entre en el IR e IB entre el 2000 al 2002, según tipo de recipientes. Durante el 2001-2002 no existieron diferencias significativas para el IR en Marzo-2001 y Diciembre-2002 entre las diez categorías de recipientes (Tabla 12). El recipiente tipo tres presentó valores de IR más altos en Agosto-2001; el recipiente tipo ocho tuvo valores más altos de IR en Diciembre-2001 y Abril-2002; finalmente el recipiente tipo nueve presentó valores más altos de IR en Agosto-2001 y Agosto2002 (Tabla 12).

Durante el 2001-2002 existieron diferencias significativas para el IB de Marzo-2001 a Diciembre-2002 entre las diez categorías de recipientes (Tabla 13). El recipiente tipo tres presentó valores de IB altos en Agosto-2001; el 

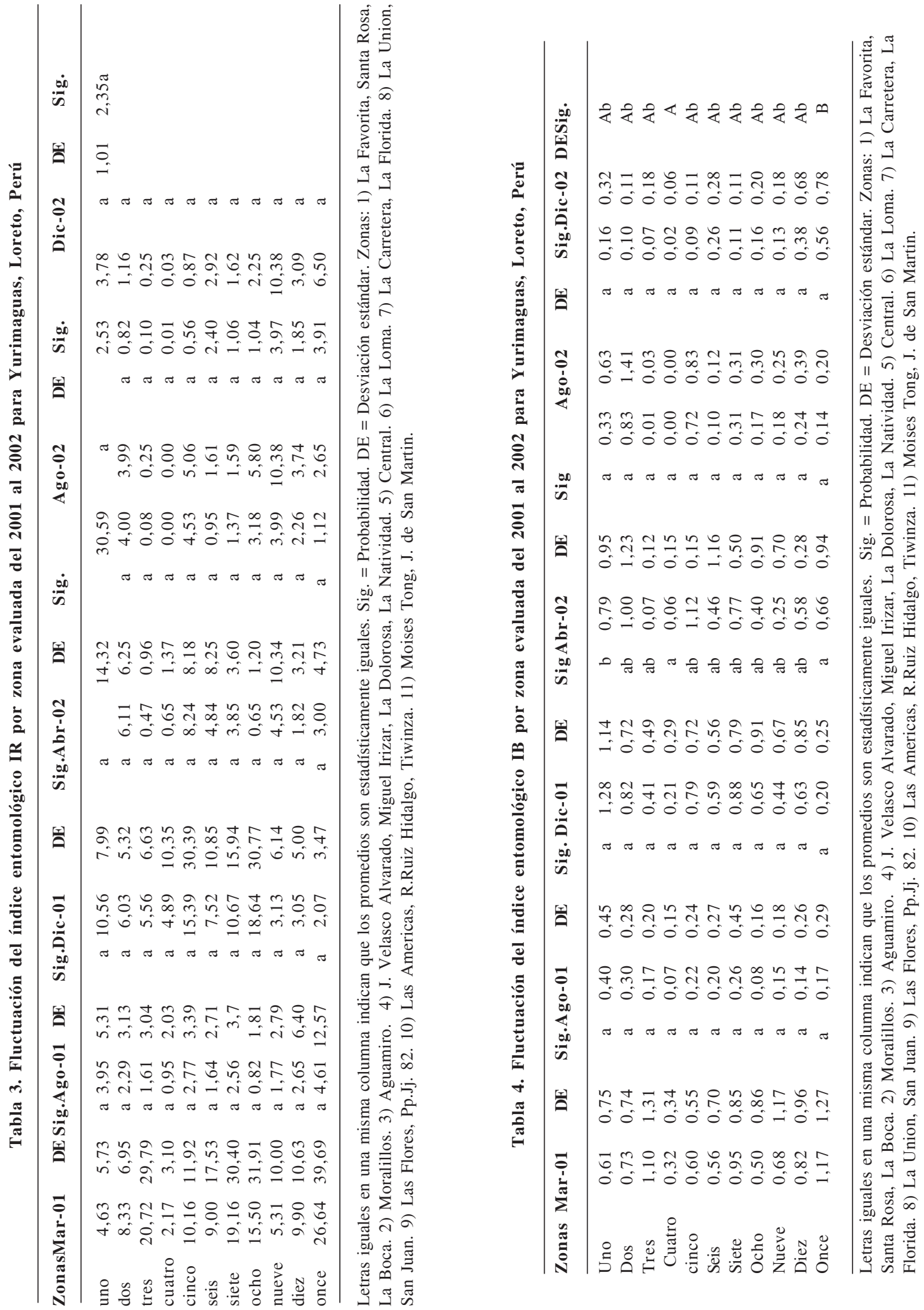
Tabla 5. Matriz de correlación de Spearman para los tres índices entomológicos entre los años 2000 al 2002

\begin{tabular}{|c|c|c|c|}
\hline & \multicolumn{3}{|c|}{ Significancia } \\
\hline & IA & & $\mathbf{I R}$ \\
\hline Correlación IA & - & 0,00 & 0,00 \\
\hline IB & 0,98 & & 0,00 \\
\hline Spearman IR & 0,94 & 0,96 & - \\
\hline
\end{tabular}

IA = Índice de infestación aédicaIB = Índice de BreteauIR = Índice de recipientes
Tabla 7. Casos de dengue entre el 2000 y el 2002 para Yurimaguas, Loreto, Perú

\begin{tabular}{lrrrrrr}
\hline Año & $\begin{array}{r}\mathbf{P C}(\mathbf{X} \\
\mathbf{n = 3 6}\end{array}$ & DE & Sig. & $\begin{array}{l}\mathbf{P C}(\mathbf{X} \\
\mathbf{n = 1 2}\end{array}$ & DE) & Sig. \\
\hline 2000 & 15,5 & 22,04 & a & 6,16 & 4,62 & a \\
2001 & 7,5 & 2,47 & a & 7,00 & 4,00 & a \\
2002 & 3,0 & 2,50 & a & 4,33 & 0,57 & a \\
\hline
\end{tabular}

Letras iguales en una misma columna indican que los promedios son estadísticamente iguales. Sig. = Probabilidad. $\mathrm{X}=$ Promedio; $\mathrm{DE}=$ Desviación estándar. $\mathrm{PC}=$ Promedio de casos mensuales.

Tabla 6. Fluctuación de los tres índices entomológicos IA, IR e IB entre el 2000 y el 2002 para Yurimaguas, Loreto, Perú

\begin{tabular}{lrrrrrrrrr}
\hline Año & IA (X & DE) & Sig. & IR(X & DE) & Sig. & IB $(\mathbf{X}$ & DE) & Sig. \\
\hline 2000 & 5,19 & 2,16 & a & 2,11 & 1,64 & a & 6,13 & 2,62 & a \\
2001 & 4,19 & 2,47 & ab & 1,03 & 0,69 & ab & 5,17 & 3,28 & ab \\
2002 & 2,76 & 2,42 & b & 0,69 & 0,70 & b & 3,45 & 3,22 & b \\
\hline
\end{tabular}

Letras iguales en una misma columna indican que los promedios son estadísticamente iguales. Sig. = Probabilidad. $\mathrm{X}=$ Promedio; $\mathrm{DE}=$ Desviación estándar.

Tabla 8. Variación de los índices entomológicos IA, IR e IB para el 2001 al 2002 entre sectores Norte y Sur de Yurimaguas, Loreto, Perú

\begin{tabular}{lcccccc}
\hline Evaluación & IA $(\mathbf{X}$ & DE $)$ & IR $(\mathbf{X}$ & DE $)$ & IB $(\mathbf{X}$ & DE $)$ \\
\hline Zona 1 a 5 & 3,50 & 2,89 & 0,98 & 0,89 & 4,47 & 3,94 \\
Zona 6 a 11 & 3,45 & 2,24 & 0,76 & 0,51 & 4,18 & 2,79 \\
\hline
\end{tabular}

Zona 1 a 5 = Sector Norte. Zona 6 a 11 = Sector Sur. Zonas: 1) La Favorita, Santa Rosa, La Boca. 2) Moralillos. 3) Aguamiro. 4) J. Velasco Alvarado, Miguel Irizar, La Dolorosa, La Natividad. 5) Central. 6) La Loma. 7) La Carretera, La Florida. 8) La Union, San Juan. 9) Las Flores, Pp.Jj. 82. 10) Las Americas, R.Ruiz Hidalgo, Tiwinza. 11) Moises Tong, J. de San Martin.

recipiente tipo seis tuvo valores más altos de IB en Marzo-2001 y Diciembre-2001; el recipiente tipo nueve presentó valores altos de IB en Marzo2001, Diciembre-2001 y Abril-2002; finalmente, el recipiente tipo diez (otros inservibles) presentó los valores más altos de IB en las seis evaluaciones del periodo 2001- 2002 (Tabla 13). La Tabla 14 mostró que no existieron diferencias en los tres IE entre el periodo seco y lluvioso, a pesar de que numéricamente la estación lluviosa en los tres casos presentó valores más altos.

El $89,98 \%$ de las casas presentaron focos positivos a A. aegypti durante el 2000 al 2002. Menos del $5 \%$ presentaron casos con recurrencia de focos positivos a mosquito de 3 a más veces (Tabla 15). La Tabla 16 nos señala que durante el 2000 al 2002, los cuatro tipos de envases que presentaron mayor frecuencia de positividad fueron: inservibles, tanque bajo, llantas y floreromacetero.

En los tres años de evaluación se realizaron 58.629 inspecciones a viviendas y 227.009 inspecciones a depósitos, encontrando un total de 3.863 depósitos positivos a A. aegypti y 2.324 casas positivas o infestadas (Tabla 17). Además se ha calculado que la proporción de depósitos potenciales por vivienda es de 5. La Tabla 18 nos muestra las viviendas y depósitos inspeccionados 


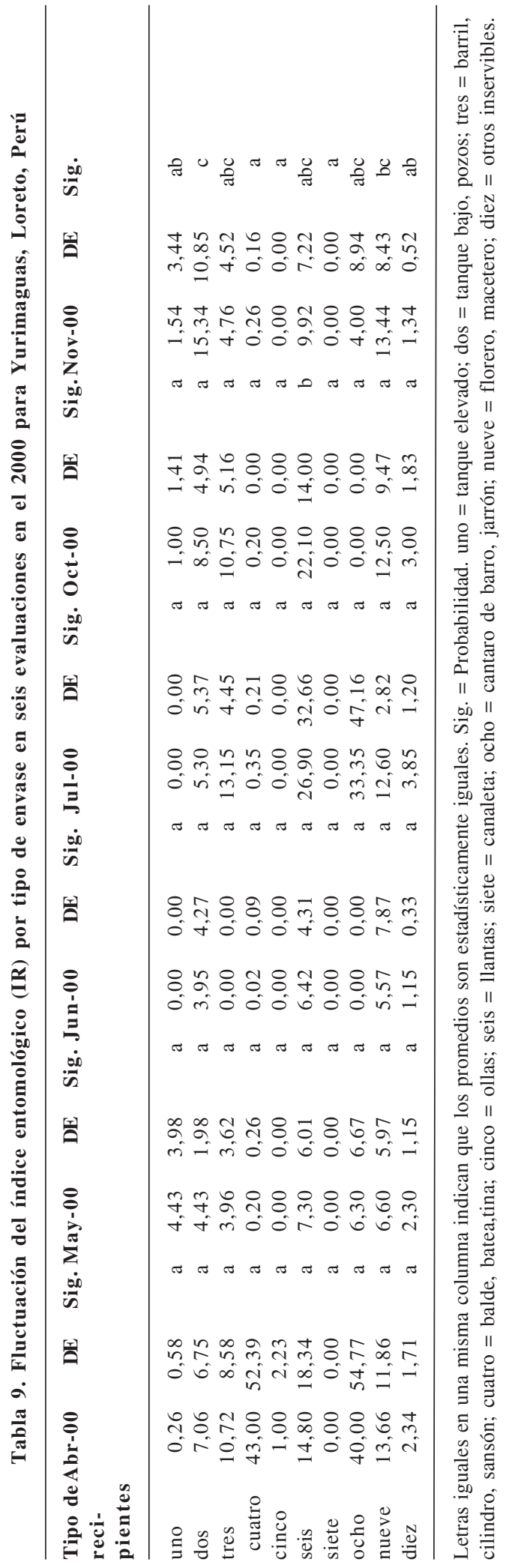

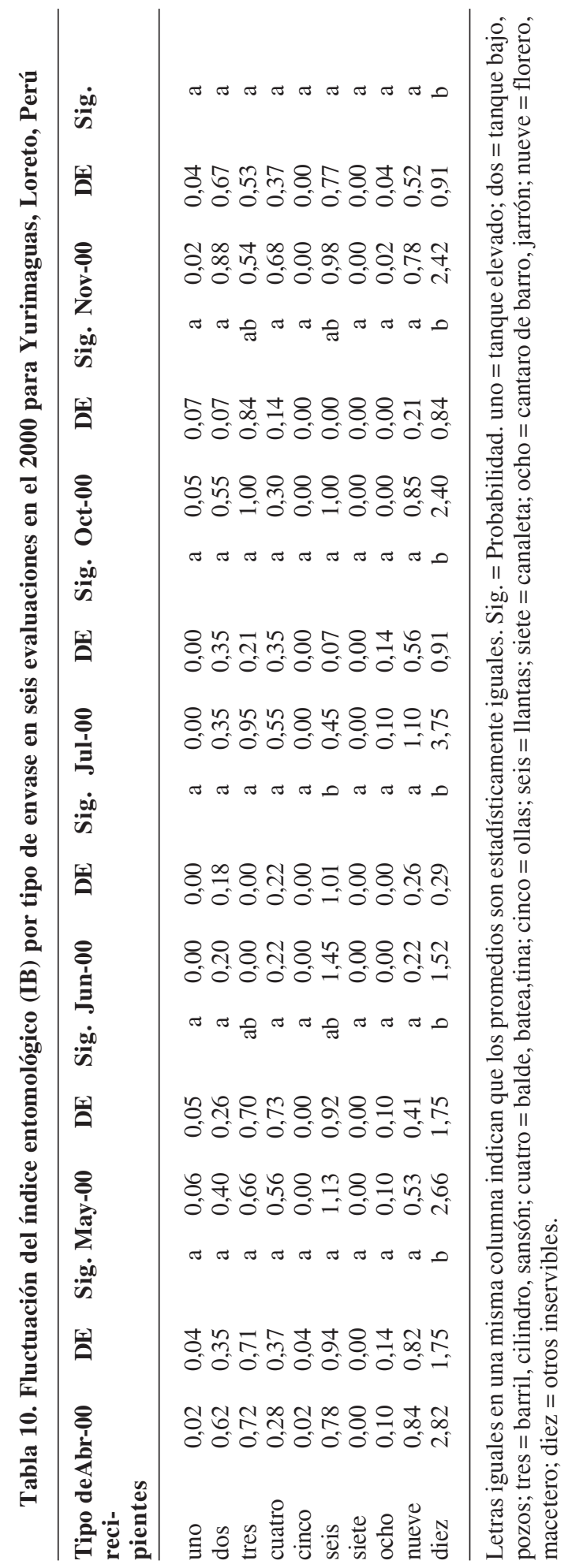


Indices larvarios de Aedes y su relación con dengue cases - W. F. Fernández y J. Iannacone

Tabla 11. Fluctuación de dos índices entomológicos: IR e IB para el 2000 al 2002 por tipo de recipiente para Yurimaguas, Loreto, Perú

\begin{tabular}{lrrrrrr}
\hline $\begin{array}{l}\text { Tipo de } \\
\text { recipiente }\end{array}$ & IR $(\mathbf{X}$ & DE) & Sig. & IB $(\mathbf{X}$ & DE) & Sig. \\
\hline uno & 1,68 & 1,67 & $\mathrm{a}$ & 0,03 & 0,08 & $\mathrm{a}$ \\
dos & 5,60 & 2,32 & $\mathrm{abc}$ & 0,41 & 0,43 & $\mathrm{c}$ \\
tres & 7,05 & 3,78 & $\mathrm{abc}$ & 0,52 & 0,48 & $\mathrm{~cd}$ \\
cuatro & 2,64 & 2.03 & $\mathrm{abc}$ & 0,34 & 0,38 & $\mathrm{bc}$ \\
cinco & 1,14 & 1,06 & $\mathrm{a}$ & 0,08 & 0,15 & $\mathrm{ab}$ \\
seis & 8,31 & 4,12 & $\mathrm{bcd}$ & 0,83 & 0,80 & $\mathrm{e}$ \\
siete & 3,02 & 2,05 & $\mathrm{abc}$ & 0,01 & 0,06 & $\mathrm{a}$ \\
ocho & 13,36 & 5,34 & $\mathrm{~d}$ & 0,05 & 0,11 & $\mathrm{a}$ \\
nueve & 10,81 & 4,48 & $\mathrm{~cd}$ & 0,71 & 0,70 & $\mathrm{de}$ \\
diez & 0,90 & 0,97 & $\mathrm{a}$ & 1,63 & 1,31 & $\mathrm{f}$ \\
\hline
\end{tabular}

Letras iguales en una misma columna indican que los promedios son estadísticamente iguales. Sig. $=$ Probabilidad. uno $=$ tanque elevado; dos = tanque bajo, pozos; tres = barril, cilindro, sansón; cuatro = balde, batea,tina; cinco = ollas; seis $=$ llantas; siete $=$ canaleta; ocho $=$ cantaro de barro, jarrón; nueve $=$ florero, macetero; diez $=$ otros inservibles .

durante los años de estudio, así como, los depósitos y viviendas infestadas. Se nota que la zona 7, presentó el mayor número de recipientes positivos (658) y la zona 2, el mayor número de casas positivas (457). La zona 8 tuvo la mayor proporción de depósitos potenciales por vivienda. Se observó una correlación lineal entre el número de depósitos y ocurrencia de casos de dengue; así como entre casas positivas y los casos de dengue.

\section{DISCUSIÓN}

Los factores responsables del resurgimiento del dengue como un problema de Salud Pública son complejos y se encuentran estrechamente asociados a cambios sociales y demográficos producidos en los últimos años ${ }^{32,33}$. El crecimiento sin precedente de la población global, asociada a la urbanización no planificada y descontrolada, especialmente en países tropicales en desarrollo (viviendas precarias, hacinamiento, deterioro en los sistemas de suministros de agua, red de alcantarillado y tratamiento de desperdicios) ha creado las condiciones ideales para el incremento del vector transmisor de la enfermedad ${ }^{34,35}$.

Las medidas de control se encaminaron como en otras regiones del país, a las campañas cívicas de recogida de inservibles, o reducción de fuentes de proliferación del vector (Tabla 17), las que han demostrado ser una buena estrategia para el control sostenible de A. aegypti en zonas urbanas en otros países ${ }^{14,36-37}$. Las acciones de control realizadas en los años 2000 al 2002 han mostrado ser efectivas en la reducción de los tres IE aédicos, a pesar de la escasa colaboración de las autoridades municipales locales ${ }^{38,39}$.

Las estrategias de control empleadas para el control de A. aegypti incluyeron campañas cívicas de recogida o y eliminación de inservibles y luego la aplicación de insecticida en los focos (Temephos, Abate ${ }^{\circledR}, 1 \%$ G) en toda la ciudad, las mismas que se han mantenido en forma sostenible por lo menos cada 4 meses ( 3 veces al año) durante los años 2001 y 2002; asimismo la aplicación de adulticidas sintéticos (Ciflutrina, Deltaplus (®) mediante fumigación UBV, cuando se incrementaban los índices aédicos y por ende el riesgo epidemiológico, con la finalidad de controlar los posibles brotes y adelantarnos al daño que podría ocasionar si el dengue se presentara (sobre 10 casos de dengue/semana); siendo evidente que durante el año 2001 y 2002 no se han reportado brotes de dengue a pesar que en nuestra Región Selva se presentaron brotes considerables en ambos años con presencia de casos de dengue hemorrágico (Tabla 17). Mientras que la vigilancia aédica se mantuvo a través de la medición de los tres índices (IA, IB, IR) con los censos (cada 4 meses) en el 2001 y 2002, las encuestas aédicas de verificación al 33\% post - tratamiento focal y encuestas con muestreos probabilísticos rápidos de vigilancia (aproximadamente al 10\%), teniendo casi los 12 meses del año índices que nos podían ayudar a tomar decisiones. También se mantuvo en vigilancia al adulto en forma esporádica (cuando 


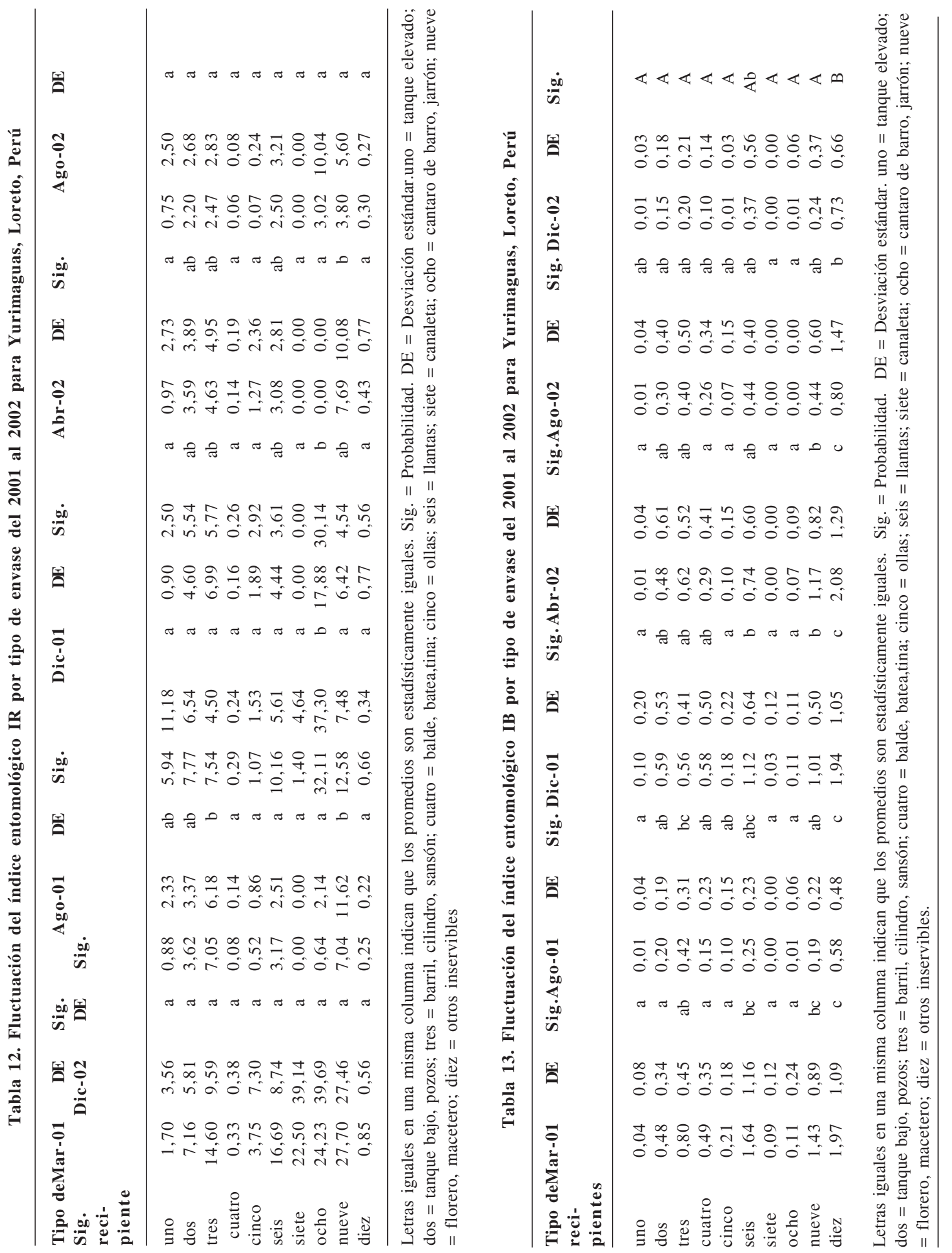


Tabla 14. Variación de los índices entomológicos IA, IR e IB entre los periododos Secos y Lluviosos en Yurimaguas, Loreto, Perú

\begin{tabular}{|c|c|c|c|c|c|c|}
\hline $\begin{array}{l}\text { Evaluación } \\
\text { Periodo }\end{array}$ & \multicolumn{2}{|c|}{ IA (X DE) } & \multicolumn{2}{|c|}{ IR (X DE) } & \multicolumn{2}{|l|}{ IB (X DE) } \\
\hline Seco & 1,75 & 1,28 & 0,85 & 4,62 & & 2,19 \\
\hline Lluvioso 4,82 & 1,71 & 1,72 & 1,57 & 5,89 & & 2,06 \\
\hline $\mathrm{t}$ & & & & & 1,03 & \\
\hline & & & & & 10 & \\
\hline significancia & & & & & 0,32 & \\
\hline
\end{tabular}

$\mathrm{t}=\mathrm{t}$ de Student. g.l. = grados de libertad. Seco = Mayo 2000, Junio 2000, Julio 2000, Octubre 2000, Agosto 2001, Agosto 2002. Lluvioso = Abril 2000, Noviembre 2000, Marzo 2001, Diciembre 2001, Abril 2002, Noviembre 2002.

Tabla 15. Frecuencia de recurrencia de casas positivas a Aedes aegypti en Yurimaguas durante el 2000 al 2002

\begin{tabular}{lcc}
\hline Recurrencia & Frecuencia & \% \\
\hline 1 & 2.180 & 83,98 \\
2 & 288 & 11,09 \\
3 & 78 & 3,00 \\
4 & 32 & 1,23 \\
5 & 9 & 0,35 \\
6 & 8 & 0,31 \\
7 & 1 & 0,04 \\
Total & 2.596 & 100 \\
\hline
\end{tabular}

los IE eran altos) o cuando el caso lo requería (antes y después de un tratamiento con adulticidas). Ha contribuyó mucho a alcanzar los resultados esperados la conformación de un Comité Bi-Sectorial (Educación - Salud) para la lucha contra el dengue en la ciudad de
Yurimaguas, el cual facilitó el componente educativo en materia de información, comunicación y educación para la salud, que a través de sus diferentes actividades sensibilizadoras logro una mayor participación de la comunidad en las acciones de control de $A$. aegypti $i^{39-41}$. La disminución de los IE y la ausencia de brotes de dengue desde el 2001 indican que las estrategias empleadas han dado buenos resultados ${ }^{37}$.

Una alta correlación lineal y positiva entre los IE, ha sido encontrada por numerosos investigadores, 17,42-44. El IB y el IR están estrechamente correlacionados y asociados con el IA, presentándose el IB con valores cercanos al IA, lo cual hace prever que ambos constituyen indicadores precisos para determinar y calificar los criterios de riesgo entomológico. Los valores del IB siempre están en rangos de calificación bajos, y muy cercanos numéricamente con los del IA, lo cual ameritaría que los números

Tabla 16. Frecuencia de recipientes positivos a Aedes aegypti en Yurimaguas durante el año 2000 al 2002

\begin{tabular}{lcccrrr}
\hline $\begin{array}{l}\text { Tipo de } \\
\text { recipiente }\end{array}$ & $\begin{array}{c}\text { Frecuencia } \\
\mathbf{2 0 0 0}\end{array}$ & $\begin{array}{c}\mathbf{\%} \\
\mathbf{2 0 0 0}\end{array}$ & $\begin{array}{c}\text { Frecuencia } \\
\mathbf{2 0 0 1}\end{array}$ & $\begin{array}{c}\boldsymbol{\%} \\
\mathbf{2 0 0 1}\end{array}$ & $\begin{array}{c}\text { Frecuencia } \\
\mathbf{2 0 0 2}\end{array}$ & $\begin{array}{c}\mathbf{\%} \\
\mathbf{2 0 0 2}\end{array}$ \\
\hline uno & 6 & 0,67 & 11 & 1,08 & 5 & 0,64 \\
dos & 208 & $2.3,32$ & 105 & 10,33 & 78 & 9,92 \\
tres & 1 & 0,11 & 132 & 12,99 & 101 & 12,85 \\
cuatro & 67 & 7,51 & 60 & 5,91 & 60 & 7,63 \\
cinco & 1 & 0,11 & 28 & 2,76 & 16 & 2,04 \\
seis & 132 & 14,80 & 187 & 18,41 & 119 & 15,14 \\
siete & 0 & 0,00 & 5 & 0,49 & 0 & 0,00 \\
ocho & 9 & 1,01 & 18 & 1,77 & 8 & 1,02 \\
nueve & 114 & 12,78 & 156 & 15,35 & 118 & 15,01 \\
diez & 354 & 39,69 & 314 & 30,91 & 281 & 35,75 \\
Total & 892 & 100 & 1.016 & 100 & 786 & 100 \\
\hline
\end{tabular}

uno = Tanque elevado; dos = tanque bajo, pozos; tres = barril, cilindro, sansón; cuatro = balde, batea, tina; Cinco = ollas; seis $=$ llantas; siete $=$ canaleta; ocho $=$ cantaro de barro, jarrón; nueve $=$ florero, macetero; diez $=$ otros inservibles . 
Indices larvarios de Aedes y su relación con dengue cases - W. F. Fernández y J. Iannacone

Tabla 17. Viviendas y depósitos inspeccionados y positivos de Aedes aegypti en la ciudad de Yurimaguas durante los años 2000 a 2002

\begin{tabular}{lccccc}
\hline Año & $\begin{array}{c}\text { Viviendas } \\
\text { Inspeccionadas }\end{array}$ & $\begin{array}{c}\text { Depositos } \\
\text { Inspeccionados }\end{array}$ & $\begin{array}{c}\text { Depositos } \\
(+) \text { de } \boldsymbol{A . \text { aegypti }}\end{array}$ & $\begin{array}{c}\text { Viviendas } \\
\text { Positivas }\end{array}$ & $\begin{array}{c}\text { Proporción de } \\
\text { Depósitos/Casa }\end{array}$ \\
\hline 2000 & 14.124 & 53.891 & 923 & 774 & 4 \\
2001 & 21.886 & 111.612 & 2.195 & 904 & 5 \\
2002 & 22.619 & 111.506 & 745 & 646 & 5 \\
Total & 58.629 & 277.009 & 3.863 & 2.324 & 5 \\
\hline
\end{tabular}

Tabla 18. Viviendas y depósitos inspeccionados y positivos de Aedes aegypti en las 11 zonas de la ciudad de Yurimaguas y su relación con los casos de dengue ocurridos, consolidado de los años 2000 a 2002

\begin{tabular}{lcccccr}
\hline Zonas & $\begin{array}{c}\text { Viviendas } \\
\text { Inspeccionadas }\end{array}$ & $\begin{array}{c}\text { Depositos } \\
\text { Inspeccionados }\end{array}$ & $\begin{array}{c}\text { Depositos } \\
\text { (+)de } \boldsymbol{A . ~ a e g y p t i}\end{array}$ & $\begin{array}{c}\text { Viviendas } \\
\text { Positivas }\end{array}$ & $\begin{array}{c}\text { Proporción de } \\
\text { Depositos/Casa }\end{array}$ & $\begin{array}{c}\text { Casos } \\
\text { Ocurridos }\end{array}$ \\
\hline uno & 3.982 & 16.216 & 339 & 196 & 4 & 13 \\
dos & 8.115 & 35.032 & 655 & 457 & 4 & 40 \\
tres & 4.556 & 23.010 & 243 & 96 & 5 & 15 \\
cuatro & 6.486 & 32.266 & 102 & 64 & 5 & 10 \\
cinco & 7.583 & 25.295 & 625 & 390 & 3 & 42 \\
seis & 9.811 & 49.660 & 640 & 373 & 5 & 51 \\
siete & 7.077 & 38.235 & 658 & 383 & 5 & 12 \\
ocho & 1.610 & 8.950 & 54 & 47 & 6 & 32 \\
nueve & 4.143 & 22.553 & 178 & 109 & 5 & 20 \\
diez & 2.702 & 13.455 & 186 & 104 & 5 & 24 \\
once & 2.564 & 12.337 & 183 & 105 & 5 & 308 \\
Total & 58.629 & 277.009 & 3.863 & 2.324 & 5 & \\
\hline
\end{tabular}

sugeridos como criterios para determinar el riesgo entomológico según OPS/OMS, 1994 deben ser revisados y adaptados para cada área específica. Se muestra una asociación entre los tres IE (IA, IB, IR), obtenidos para la ciudad en forma general, con la ocurrencia de los casos de dengue. Sin embargo no se ha encontrado esta misma correlación entre los índices obtenidos para cada zona con la ocurrencia de casos en la misma, este mismo acontecimiento ha sido registrado en Malasia ${ }^{24}$, en donde se notificaron casos en zonas con IE bajos. En Yurimaguas se podría explicar por la escasa distancia que existe entre una zona y la otra lo que permiten que la comunicación, el transporte y el flujo y reflujo de personas entre ellas sea dinámica ${ }^{45}$. La transmisión y la presencia de casos pueden darse indistintamente en cualquiera de las zonas, aun cuando los IE estén bajos. Por lo que no se debe centrar la vigilancia y control sólo en aquellas zonas en donde los IE estén elevados. Por último, zonas que presentan bajos IE no necesariamente tendrá una menor incidencia de casos.
Los principales sitios de cría de A. aegypti son mantenidos por las prácticas humanas ${ }^{46}$. En Yurimaguas, los recipientes inservibles han demostrando tener una alta probabilidad de ser preferidos por los vectores de tal manera que el control y la conducta poblacional deben incidir con más énfasis en campañas de difusión y educación sanitaria y ambiental ${ }^{42}$. Sin embargo, por la dinámica de los focos o recipientes positivos que se han presentado en estos tres años de estudio, la vigilancia y el control debe estar orientado a todos los tipos de recipientes y no únicamente a los inservibles.

Las campañas de difusión en educación para el control del dengue a través del Comité Bisectorial (Educación - Salud) en centros educativos, con concursos, conferencias, capacitaciones en talleres y discusiones, al igual que la difusión por los medios de comunicación masiva han contribuido a reducir los índices aédicos en la ciudad tal como ha sido demostrado previamente ${ }^{43}$.

Se ha demostrado que es de suma importancia 
la participación conjunta de la comunidad en las acciones de control de A. aegypti a través de la eliminación de los depósitos desechados ${ }^{15,27}$. En Yurimaguas también se está evidenciando que la participación comunitaria en las campañas cívicas de recoger y eliminar los inservibles están contribuyendo, en mayor o menor grado, al control del dengue en la ciudad, por lo que, se debe incluir en estas campañas en forma periódica ya que ayudan disminuir el riesgo. Asimismo, debe darse énfasis a la limpieza de los recipientes como un requisito más para prevenir la formación de criaderos ${ }^{47}$.

\section{RESUMEN}

Se realizó un estudio sobre el comportamiento poblacional de Aedes aegypti en su fase larvaria, para explicar sus fluctuaciones a través de tres indicadores entomológicos (IE) y su relación con los casos de dengue en la ciudad de Yurimaguas, Loreto, Perú. Se censaron las viviendas positivas a través de tres IE: \{Índice de Infestación domiciliaria o también llamado Índice aédico (IA), Índice de recipientes (IR) e Índice de Breteau (IB)\} y los tipos de recipientes más comunes en 11 zonas de la ciudad de Yurimaguas durante el 2000 al 2002. Se encontró que los tres IE presentaron diferencias entre las doce evalua-ciones realizadas durante el 2000 al 2002. En los tres IE se ha notado una tendencia a la disminución de Abril-2000 a Diciembre-2002. El IA disminuye en un 46,82\%; el IR en un $67,29 \%$ y IB disminuye en $43,71 \%$. Los casos de dengue registrados del 2000 al 2002 disminuyeron en $80,6 \%$. No se observaron diferencias entre las 11 zonas de la ciudad para IA, IR e IB. Los IA, IB e IR estuvieron altamente correlacionados lineal y positivamente durante el periodo 2000 al 2002. Sólo el IA y los casos de dengue, se encuentran correlacionados linealmente. En algunas evaluaciones del 2000 al 2002 existieron diferencias significativas para el IR e IB entre las diez categorías de recipientes. Se notaron valores altos para las cuatro categorías siguientes: 10 (inservibles), 9 (florero y macetero), 6 (llantas), tres (barril, cilindro, sansón). Se evaluaron las variaciones de los IE durante los periodos secos y lluviosos.

\section{REFERENCIAS}

1.- HARWOOD R F, JAMES M T. Entomología Médica y Veterinaria. Uteha Noriega Ed. México1993; 615 p.
2.- NATHAN M B, KNUDSEN A B. Aedes aegypti infestation characteristics in several Caribbean countries and implications for integrated community based control. J Am Mosq Control Assoc 1991; 7: 400-4.

3.- CHADEE D. Aedes aegypti surveillance in Tobago, West Indies (1983-88). J Am Mosq Control Assoc 1990; 6: 148-50.

4.- CLARK G G. Situación Epidemiológica del Dengue en América. Desafíos para su Vigilancia y Control. Rev Salud Pub Mex 1995; 37: 5-20.

5.- THAVARA U, TAWATSIN A, CHANSANG $\mathrm{C}$ et al. Larval occurrence, oviposition behavior and biting activity of potential mosquito vectors of dengue on Samui Island, Thailand. J Vector Ecol 2001; 26: 172-80.

6.- ESPINOZA GF,HERNÁNDEZSCM, COLLCR. Factores que modifican los índices larvarios de Aedes aegypti en Colima, México. Rev Pan Salud Pub 2001; 10: 6-12.

7.- MINISTERIO DE SALUD (MINSA) - DIRECCIÓN GENERAL DE SALUD AMBIENTAL (DIGESA). Manual de Campo para la Vigilancia entomológica. Lima, Perú. 2002. 140 pp.

8.- DEGALLIER N, TEIXEIRA J M, VILARINHOS P D et al. First isolation of dengue 1 virus from Aedes aegypti in Federal District, Brazil. Rev Soc Bras Med Trop 2000; 33: 95-6.

9.- ALVES M C, DA SILVA N N. Simplifying the sampling method for evaluating the larval density of Aedes aegypti in Sao Paulo State, Brazil. Rev Saude Pub 2001; 35: 467-73.

10.- HANNA J N, RITCHIE S A, PHILLIPS D A et al. An epidemic of dengue 3 in far north Queensland, 19971999. Med J Australian 2001; 174: 178-82.

11.- TIBAIRE M M. Actualización en dengue. Parte I. Rev Soc Venezolana Microbiol 2001; 21: En www.scielo.org.ve/scielo.php?script $=$ sci_arttext\&pid= $\mathrm{S} 1315-25562001000100007 \& \mathrm{Ing}=\mathrm{es} \& \mathrm{nrm}=$ iso. Leído el 17 de Febrero del 2004.

12.- BISSET J A, GONZÁLEZ B, MENDIZÁBAL M E et al. La abundancia larval de mosquitos urbanos durante la campaña de erradicación de Aedes aegypti (Linnaeus 1762) y del dengue en Cuba. Rev Cub Med Trop 1985; 37: 161-8

13.- ARMADA G A, FIGUEREDO R. Ordenamiento del medio en el programa de erradicación de Aedes aegypti, Cuba 1987. Bol Of Sanit Panam 1987; 102: 237-44.

14.- CHIARAVALLOTI N F, DA COSTA A I, MOURA M $S$ et al. An evaluation of municipal actions in the control of dengue vectors in the region of Sao Jose do Rio Preto, Sao Paulo, 1989 to 1995. Rev Soc Bras Med Trop 1999; 32: 357-62.

15.- WANG C H, CHANG N T, WU H H, HO C M. Integrated control of the dengue vector Aedes aegypti in Liu-Chiu village, Ping-Tung County, Taiwan. J Am Mosq Control Assoc 2000; 16: 93-99. 2001. Rev Per Epidemiol 2002; 10. En:

16.- URIBE J L. El problema del control de Aedes aegypti en América. Bol Of Sanit Panam 1983; 94: 473-81.

17.- SENG C M, JUTE N. Breeding of Aedes aegypti (L.) and Aedes albopictus (Skuse) in urban housing of Sibu town, Sarawak. Southeast Asian J Trop Med Public Health 1994; 25: 543-8.

18.- ROMERO V C M, WHEELER J G, FALCONAR A K. An inexpensive intervention for the control of larval 
Aedes aegypti assessed by an improved method of surveillance and analysis. J Amer Mosq Control Assoc 2002; 18: 40-6.

19.- PHILLIPS I, NEED J, ESCAMILLA J et al. Primer brote de dengue documentado en la Región Amazónica del Perú. Bol Of. Sanit Panam 1993; 114: 513-9.

20.- SEVILLA A C, CACERES A G, VAQUERIZO A et al. Reappearance of Aedes aegypti (Diptera: Culicidae) in Lima, Perú. Mem Inst Oswaldo Cruz 2001; 96: 657-8.

21.- MARQUETTI M C, GONZALES D, AGUILERA L, NAVARRO A. Indices ecológicos en el sistema de vigilancia de Aedes aegypti (Diptera: Culicidae) en Cuba. Rev Cub Med Trop 1999; 51: 79-82.

22.- TENG H J, WU Y L, LIN T H J. Mosquito fauna in water-holding containers with emphasis on dengue vectors (Diptera: Culicidae) in Chungho, Taipei County, Taiwan. Med Entomol 1999; 36: 468-72.

23.- CHADEE D D, RAHAMAN A. Use of water drums by humans and Aedes aegypti in Trinidad. J Vector Ecol 2000; 25: 28-35.

24.- SULAIMAN S, PAWANCHEE Z A, ARIFIN Z, WAHAB A. Relationship between Breteau and House indices and cases of dengue/dengue hemorrhagic fever in Kuala Lumpur, Malaysia. J Am Mosq Control Assoc 1996; 12: 494-6.

25.- IANNACONE J A, ALVARIÑO L, MORENO R et al. Culícidos (Diptera) del río Chillón y sectores adyacentes de la Provincia Constitucional del Callao, Perú, durante el Niño 1997-1998. Acta Ent Chilena 2000; 24: 51-60.

26.- TUN-LIN W, KAY B H, BARNES A, FORSYTH S. Critical examination of Aedes aegypti indices: correlations with abundance. Am J Trop Med Hig 1996; 54: 543-7.

27.- SHARMA S K, PADHAN K, RATH Y, RAO S K. Observations on the breeding habitat of Aedes species in the steel township, Rourkela. J Comm Disease 2001; 33: $28-35$.

28.- STONE A. Culicidae. En: Manual of Neartic Diptera. Volume 1. Mc Alpine J F, Peterson B V, Shewell G E, Teskey H J, Vockeroth J R, Wood D M (Eds). Research Brach Agriculture Canada. Monograph 27. 1981. pp. 341-50.

29.- MERRITT R W, CUMMINS K W. An Introduction to the aquatic Insects of North America. Kendall. unt Publishing Co. Dubuque. I.A. U.S.A. 1984. 650 pp.

30.- BALTA L R. Guía Práctica para la identificación de Aedes aegypti. Ministerio de Salud - Instituto Nacional de Salud. Serie de Guías Entomológicas $\mathrm{N}^{\circ}$ 2. Lima, Perú. 1997. 24 pp.

31.- LUDUENA A F F, GORITA D E. The biology of Aedes (Ochlerotatus) albifasciatus Macquart, 1838 (Diptera: Culicidae) in Central Argentina. Mem Inst Oswaldo Cruz 1995; 90: 463-8.

32.- VALDÉS L, GUZMÁN M, KOURÍ G et al. La epidemiología del dengue y del dengue hemorrágico en Santiago de Cuba, 1997. Rev Pan Salud Pub 1999; 6: 16-24.

33.- ALFARO M, LUNA M, NOVOA Y et al. Factores fundamentales de exposición al Dengue según escenarios geo-epidemiológicos en Trujillo Metropolitano. AbrilMayo 2001. Rev Peru Epidemiol 2002 10: En: sisbib.unmsm.edu.pe/BVRevistas/epidemiologia/ vol10_N7_2002/factores_dengue.htm Leído el 17 de febrero del 2004.

34.- MARQUETTI M C, CARUS F, AGUILERA L, NAVARRO A. Influencia de factores abióticos sobre la incidencia de Aedes aegypti en el municipio 10 de Octubre de Ciudad de La Habana, 1982-1992. Rev Cub Med Trop 1995; 47: 88-92.

35.- BUENO C, CARRANZA J, LLONTOP A, VELA F. Dengue en San Martín: seis años de experiencias. Hojas Amazónicas de Salud Pública 2 (DIRES SAN MARTIN, MINSA). 1998

36.- ORGANIZACIÓN PANAMERICANA DE LA SALUD (OPS). Dengue y dengue hemorrágico en las Américas: guías para su prevención y control. Washington, DC: OPS, Pub. Científica 1995; 548: 1-109.

37.- DANIS L R, RODRÍGUEZ M H, HERNÁNDEZ ÁVILA M. Gender-related family head schooling and Aedes aegypti larval breeding risk in Southern México. Rev Salud Pub Mex 2002; 44: 237-52.

38.- MARQUETTI M C, CARUS F, AGUILERA L et al. Comportamiento del programa de erradicación de Aedes aegypti en 2 municipios de Ciudad de La Habana, 19901992. Rev Cub Med Trop1996; 48: 174-7.

39.- DE CASTRO G A. Medidas dos niveis de infestacao urbana para Aedes (Stegomyia) aegypti e Aedes (Stegomyia) albopictus em programa de vigilancia entomológica. IESUS 1998; 7: 49-57.

40.- DE LA CRUZ A M, FIGUEROA D, CHACON L et al. Conocimientos, opiniones y prácticas sobre Aedes aegypti. Rev Cub Med Trop 1999; 51: 135-7.

41.- DE LA CRUZ A M, MESA A, SAN MARTIN J L. La comunidad y el control de Aedes aegypti: percepción y comportamiento respecto al larvicida abate. Rev Cub Med Trop 2001; 53: 44-7.

42.- LLOYD L S, WINCH P, ORTEGA-CANTO J, KENDALL C. Results of a community-based Aedes aegypti control program in Merida, Yucatan, Mexico. Am J Trop Med Hig 1992; 46: 635-42.

43.- SWADDIWUDHIPONG W, LERDLUKANAVONGE P, KHUMKLAM P et al. A survey of knowledge, attitude and practice of the prevention of dengue hemorrhagic fever in an urban community of Thailand. Southeast Asian J Trop Med Pub Health 1992a; 23: 207-11.

44.- SWADDIWUDHIPONG W, CHAOVAKIRATIPONGC, NGUNTRA $P$ et al. Effect of health education in community participation in control of dengue hemorrhagic fever in an urban area of Thailand. Southeast Asian J Trop Med Pub Health 1992b; 23: 200-6.

45.- FAURAN P, LE CHANTILLY N. . Prediction and prevention of dengue epidemics. Bull Soc Pathol Exoc 1996; 89: 123-6.

46.- YEBAKIMA A. Control of Aedes aegypti in Martinique: contribution of entomology studies. Bull Soc Pathol Exoc 1996; 89: 161-2.

47.- FORATTINI O P, KAKITANI I, SALLUM M A, DE REZENDE L. Productivity of a breeding place of Aedes albopictus in an urban environment. Rev Saude Pub 1997; 31: 545-55.

Agradecimientos: Agradecemos a los laboratorios del Hospital de Apoyo Yurimaguas - Dirección de Salud Ambiental y Dirección de Epidemiología, Red de Servicios de Salud de Alto Amazonas. Al personal del Laboratorio de Entomología y del Programa de Lucha contra el Dengue (Vigilancia y Control) de la Dirección de Salud Ambiental: M. T. Santa Cruz, F. Carvajal, F. Fernández, J. Tello, E. Rodríguez y a todos los técnicos e inspectores de campo por su colaboración en la ejecución de este proyecto de Investigación. Este trabajo fue presentado en el XVI Congreso Latinoamericano de Parasitología, La Paz- Bolivia, 1 al 3 de Octubre 2003. 